\title{
The asymmetry of female meiosis reduces the frequency of inheritance of unpaired chromosomes
}

\author{
Daniel B Cortes ${ }^{1}$, Karen L McNally ${ }^{1}$, Paul E Mains ${ }^{2}$, Francis J McNally ${ }^{1 *}$ \\ ${ }^{1}$ Department of Molecular and Cellular Biology, University of California, Davis, Davis, \\ United States; ${ }^{2}$ Department of Biochemistry and Molecular Biology, University of \\ Calgary, Calgary, Canada
}

\begin{abstract}
Trisomy, the presence of a third copy of one chromosome, is deleterious and results in inviable or defective progeny if passed through the germ line. Random segregation of an extra chromosome is predicted to result in a high frequency of trisomic offspring from a trisomic parent. Caenorhabditis elegans with trisomy of the $\mathrm{X}$ chromosome, however, have far fewer trisomic offspring than expected. We found that the extra $X$ chromosome was preferentially eliminated during anaphase I of female meiosis. We utilized a mutant with a specific defect in pairing of the $X$ chromosome as a model to investigate the apparent bias against univalent inheritance. First, univalents lagged during anaphase I and their movement was biased toward the cortex and future polar body. Second, late-lagging univalents were frequently captured by the ingressing polar body contractile ring. The asymmetry of female meiosis can thus partially correct pre-existing trisomy. DOI: 10.7554/eLife.06056.001
\end{abstract}

*For correspondence: fjmcnally@ ucdavis.edu

Competing interests: The authors declare that no competing interests exist.

Funding: See page 18

Received: 12 December 2014 Accepted: 03 April 2015

Published: 07 April 2015

Reviewing editor: Anthony A Hyman, Max Planck Institute of Molecular Cell Biology and Genetics, Germany

(c) Copyright Cortes et al. This article is distributed under the terms of the Creative Commons Attribution License, which permits unrestricted use and redistribution provided that the original author and source are credited.

\section{Introduction}

During female meiosis, a G2 oocyte containing four genome copies undergoes two asymmetric cell divisions depositing one genome in a single haploid egg, while the other three genomes are segregated into polar bodies. These divisions are mediated by meiotic spindles that are asymmetrically positioned against the oocyte cortex with the pole-to-pole axis of the spindle perpendicular to the cortex. Both the inheritance of only one of the four genome copies and the distinct perpendicular positioning of the meiotic spindle are remarkably conserved among animal phyla suggesting a selective advantage (Maro and Verlhac, 2002; Fabritius et al., 2011a; Maddox et al., 2012).

Several advantages of asymmetric meiosis have been suggested previously, yet none are applicable to all animals. Asymmetric meiotic spindle positioning maximizes the volume of a single egg, helps prevent interference with the meiotic spindle by the sperm aster (McNally et al., 2012), and preserves predetermined embryonic polarity gradients. Here, we suggest a previously unrecognized advantage of asymmetric meiosis, the ability of meiotic spindles to correct trisomy by preferentially depositing the extra chromosome copy into a polar body.

Accurate segregation of homologous chromosomes to opposite spindle poles depends on a physical attachment, or chiasma, between homologous chromosomes. A chiasma consists of a crossover, which holds the two homologous chromosomes together in a bivalent so that kinetochores can be properly oriented toward opposite poles (Moore and Orr-Weaver, 1998; Miller et al., 2013). When a chiasma does not form, univalent chromosomes may maintain sister cohesion and move to poles independent of their homologs at anaphase I as can occur in Saccharomyces cerevisiae (Buonomo et al., 2000). If a univalent chromosome biorients, loses cohesion, and segregates sister chromatids at anaphase I (e.g., Nicklas and Jones, 1977; Lemaire-Adkins and 
eLife digest Inside cells, DNA is found packaged into structures called chromosomes. Most human and animal cells contain two sets of chromosomes, one inherited from each parent.

Chromosomes from one set pair up with the equivalent chromosome from the other set. However, egg and sperm cells only contain one copy of each chromosome, so that when the egg is fertilized, the resulting cell again has two sets of chromosomes. If there are either more or fewer than two copies of a chromosome in the fertilized cell, this can cause birth defects and conditions such as Down syndrome.

An egg cell develops from a cell called an oocyte via a process called meiosis. The oocyte first duplicates its DNA so that it contains four copies of each chromosome. The oocyte then divides, and the resulting cells divide again, to produce four cells that each contains one copy of each chromosome. Only one of these cells is an egg cell: the other three are called polar bodies, and these normally self-destruct.

The tiny roundworm $C$. elegans is a model organism used to study meiosis. Worms can be hermaphrodites or males; the hermaphrodites normally have a pair of ' $\mathrm{X}$ ' sex chromosomes. However, sometimes problems with meiosis can produce hermaphrodite worms with three $X$ chromosomes in each of their cells. In these cells, two of the $X$ chromosomes pair with each other as normal, and one $\mathrm{X}$ chromosome remains unpaired.

Cortes et al. examined meiosis in mutant worms that had an extra copy of the $\mathrm{X}$ chromosome by marking all the chromosomes with a fluorescent tag. This allowed the movement of the chromosomes to be tracked through images taken using a microscope. This revealed that an unpaired $\mathrm{X}$ chromosome moves more slowly than a normal paired set. Furthermore, the unpaired chromosomes tend to move toward the region of the oocyte that will develop into a polar body. Thus, when the oocyte divides, the unpaired chromosomes are placed in the polar body and eliminated. This mechanism improves the chance that the correct number of chromosomes will end up in the egg cell.

Women with three $\mathrm{X}$ chromosomes are often fertile and in most cases produce normal offspring. Further work is needed to see whether human oocytes remove extra chromosomes by a mechanism similar to that seen in the roundworms.

DOI: $10.7554 /$ eLife. 06056.002

Hunt, 2000; Kouznetsova et al., 2007), the resulting single chromatid will segregate randomly at anaphase II. Random segregation of homologs at anaphase I or single chromatids at anaphase II should result in equal frequencies of haplo and diplo ova in the case of a trisomy (Figure 1A) and equal frequencies of nullo and diplo ova in the case of a crossover failure.

Deviations from random segregation are suggested by observations of $X$ chromosomes in Caenorhabditis elegans. In C. elegans, the single unpaired X chromosome from an XXX mother is inherited with unexpectedly low frequency with twice as many haploX ova produced as diploX ova (Hodgkin et al., 1979). HIM-8 is a zinc finger protein that binds to specific DNA sequences that are enriched on the $X$ chromosome. him- 8 mutants have a pairing defect that is completely specific for the $X$ chromosome, resulting in two $X$ univalents and five autosomal bivalents in $95 \%$ of diakinesis oocytes (Phillips et al., 2005). If the two $X$ univalents segregated randomly, him- 8 mutants would be expected to produce equal frequencies of nulloX ova and diploX ova. However, Hodgkin et al. (1979) demonstrated a fivefold preponderance of nulloX ova over diploX ova in him-8. Using sex-reversed him- 8 XX males, these authors showed the opposite effect in spermatogenesis. Rather than producing the $50 \%$ haploX, $25 \%$ diploX, 25\% nulloX sperm expected from random segregation, him- 8 XX males produced $86 \%$ haploX, $3 \%$ diploX, $11 \%$ nulloX sperm, indicating symmetric distribution of univalents during male meiosis. Thus, achiasmate maternal $X$ chromosomes are inherited with unexpectedly low frequency in worms.

Five mechanisms might reduce the frequency of trisomic offspring from trisomic or him-8 mothers. First, trisomic embryos might die during embryonic development resulting in undercounting of XXX offspring. This is unlikely in C. elegans because both XXX and him-8 mutant mothers produce a very low frequency of dead embryos (Hodgkin et al., 1979; Supplementary file 1). A second possibility is that mitotic non-disjunction in the $X X X$ mother results in a mosaic 


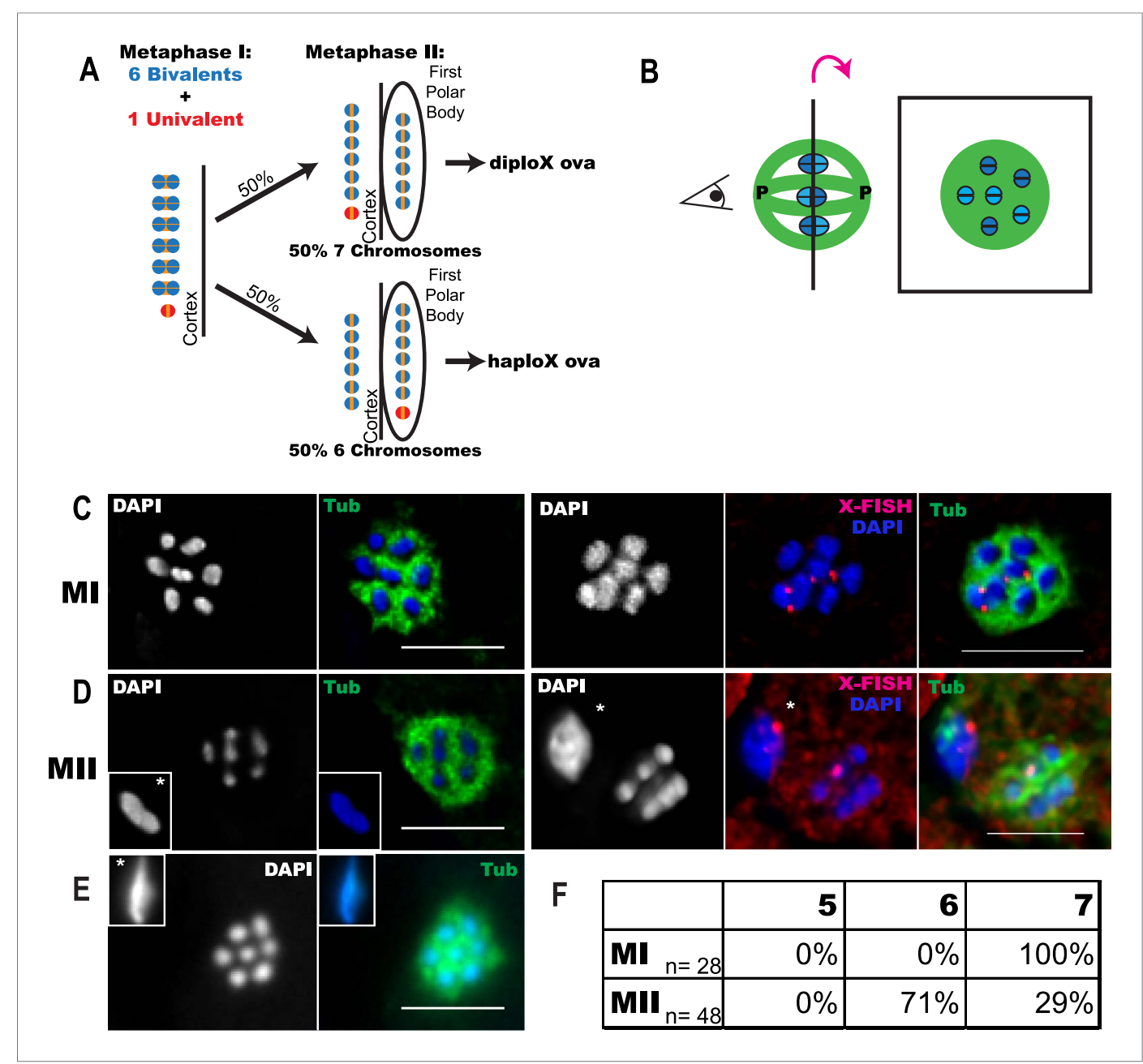

Figure 1. Trisomy correction during meiosis I. (A) Illustration showing expected outcomes of female meiosis in XXX wild-type worms, assuming the extra univalent $X$ (red) does not lose cohesion (yellow) between sister chromatids during anaphase I and assuming random segregation. (B) Illustration of a spindle with chromosomes at the metaphase plate with poles marked ' $P$ ' (left) and a projection of the cross-sectional view down the pole-to-pole axis at the metaphase plate (right). (C-E) Z projections of fixed meiotic embryos viewed down the pole-to-pole spindle axis. Meiotic embryos from XXX wild-type mothers were stained with DAPI and anti-tubulin antibody. (C) Metaphase I spindles with 7 chromosomes; right images of X-fluorescence in situ hybridization (FISH) show two X chromosomes on the spindle. See also Supplementary file 2. (D) Metaphase II spindles with 6 chromosomes; right images of X-FISH show one X on the spindle and 2-3 foci in the polar body. See also Supplementary file 3. (E) Metaphase II spindle with 7 chromosomes. (F) Frequency of each spindle class among the progeny of XXX wild-type mothers. Insets show polar bodies, marked by asterisks, which were used to identify metaphase II spindles. Bar $=5 \mu \mathrm{m}$. DOI: 10.7554/eLife.06056.003

gonad that contains both diploX and triploX oocytes. Selective apoptosis of XXX germ line cells (Bhalla and Dernburg, 2005) would then enrich for XX germ line cells. This does not contribute to the segregation bias in C. elegans, as the most mature diakinesis oocytes in him-8 and wild-type XXX worms have 7 rather than 6 DAPI-staining bodies (Phillips et al., 2005; this study). A third possibility is that a univalent present during metaphase I or a single chromatid present during metaphase II would be broken or otherwise degraded during anaphase. A fourth possibility is that many XXX progeny look normal because of the stochastic nature of dosage compensation and thus are undercounted. A fifth possibility is that univalent chromosomes present at metaphase I are preferentially placed in the first polar body. Here, we demonstrate that indeed biased deposition of univalent $X$ chromosomes into the first polar body reduces the frequency of trisomic zygotes resulting from oocytes with unpaired $X$ chromosomes. 


\section{Results}

\section{XXX wild-type oocytes preferentially lose the achiasmate $X$ chromosome between metaphase I and metaphase II}

Elimination of the extra chromosome from an oocyte starting with a trisomy would result in rescue to a euploid state. It has previously been shown that $C$. elegans $X X X$ wild-type oocytes have a paired bivalent $X$ and an unpaired univalent $X$ chromosome in pachytene (Goldstein, 1984). We picked wildtype XXX adult hermaphrodites from the progeny of an XXX strain (AV494, Mlynarczyk-Evans et al., 2013) based on their characteristic dumpy morphology as described by Hodgkin et al. (1979). Meiotic embryos from XXX mothers were fixed and stained for microtubules and DNA. Chromosomes are well separated by bundles of microtubules during $C$. elegans female meiotic metaphase. This unique morphology facilitates counting of individual chromosomes on the metaphase plate when viewed down the pole-to-pole axis of the spindle (Figure 1B). We found that $100 \%$ of metaphase I meiotic embryos from XXX wild-type worms had 7 DAPI-staining bodies on the spindle (Figure $1 C, F$ ), consistent with 6 bivalents and a single univalent $X$. Two chromosomes were labeled with an $\mathrm{X}$-specific fluorescence in situ hybridization (FISH) probe in these spindles (Figure 1C, Supplementary file 2). This result shows that a mosaic gonad resulting from mitotic nondisjunction cannot explain the low frequency of $X X X$ offspring from $X X X$ worms. If the univalent segregated randomly during anaphase $I$, $50 \%$ of metaphase II spindles should have 6 DAPI-staining bodies ( 6 bivalents) and $50 \%$ should have 7 DAPI-staining bodies ( 6 bivalents and 1 univalent). Instead, $71 \%$ of metaphase II spindles contained only 6 DAPI-staining bodies and only $29 \%$ contained 7 DAPI-staining bodies (Figure 1D-F). These frequencies match the 2:1 ratio of $X$ to $X X$ ova previously interpreted from genetic studies (Hodgkin et al., 1979) and are significantly different than the $50 \%$ expected from random segregation (onetailed $p=0.004$, Pearson's chi-squared test). This result eliminates the possibilities that XXX mothers have many $X X X$ offspring that are undercounted due to incomplete penetrance of the $X X X$ dumpy phenotype or that hermaphrodite nulloX sperm contributes significantly to the low frequency of XXX self-progeny. The finding that all assayed metaphase I spindles had 7 chromosomes also indicates that our method of identifying XXX worms as dumpy individuals is accurate and the high frequency of metaphase II spindles with 6 chromosomes is not a result of misidentifying diploid worms as XXX worms. FISH with an X-specific probe revealed that in $6 / 6$ metaphase II embryos with only 6 DAPIstaining bodies, a single hybridization signal was present in the spindle and 2-3 hybridization signals were present in the polar body (Figure 1D; Supplementary file 3). Because only a single $X$-hybridization signal was observed in the first polar body in $5 / 5$ spindles from diploids, these results demonstrate that single $\mathrm{X}$ univalents are deposited in the first polar body with greater than $50 \%$ frequency.

\section{$\mathrm{X}$ and $\mathrm{V}$ univalents are frequently deposited in the first polar body}

To further investigate the mechanism leading to preferential loss of univalents during meiosis I, we utilized him-8 worms as a more tractable model. It has previously been shown that diakinesis stage him-8 oocytes have 5 autosomal bivalents and two X univalents (Phillips et al., 2005). If segregation of the two $X$ univalents was random, these worms should produce equal numbers of nulloX and diploX ova. Instead, him-8 mutants produce a fivefold higher frequency of nulloX ova over diploX ova (Hodgkin et alo, 1979), indicating that both maternal $X$ univalents are lost at some time after diakinesis in a large fraction of embryos. To determine when maternal $X$ univalents are preferentially lost, we imaged both live embryos within him-8 worms expressing GFP::tubulin and mCherry::histone (Figure 2A-E) and also fixed embryos stained with DAPI and anti-tubulin antibodies (Figure 2F-J). We assayed the number of chromosomes (defined here as DAPI-staining or mCherry:histone-positive bodies that would include univalents and bivalents) present at metaphase of meiosis I and II (Figure 2K). At meiosis I metaphase, 7 chromosomes were present in $96 \%$ of him- 8 embryos (Figure $2 \mathrm{~B}, \mathrm{~K}$ ), with the remainder having 6 chromosomes. If the two univalents segregated randomly without losing cohesion, $25 \%$ of metaphase II spindles would be expected to have 5 autosomes and no $X, 50 \%$ would have 5 autosomes and $1 X$, and $25 \%$ would have 5 autosomes and $2 X$ chromosomes. Instead, $40 \%$ of him- 8 metaphase II embryos had 5 chromosomes, $55 \%$ had 6 chromosomes, and only $5 \%$ had 7 chromosomes (Figure $2 K$ ). These frequencies differ significantly from those expected from unbiased segregation (chi-square test, two-tailed $p<0.0001$ ), closely match the ratio of nulloX to 

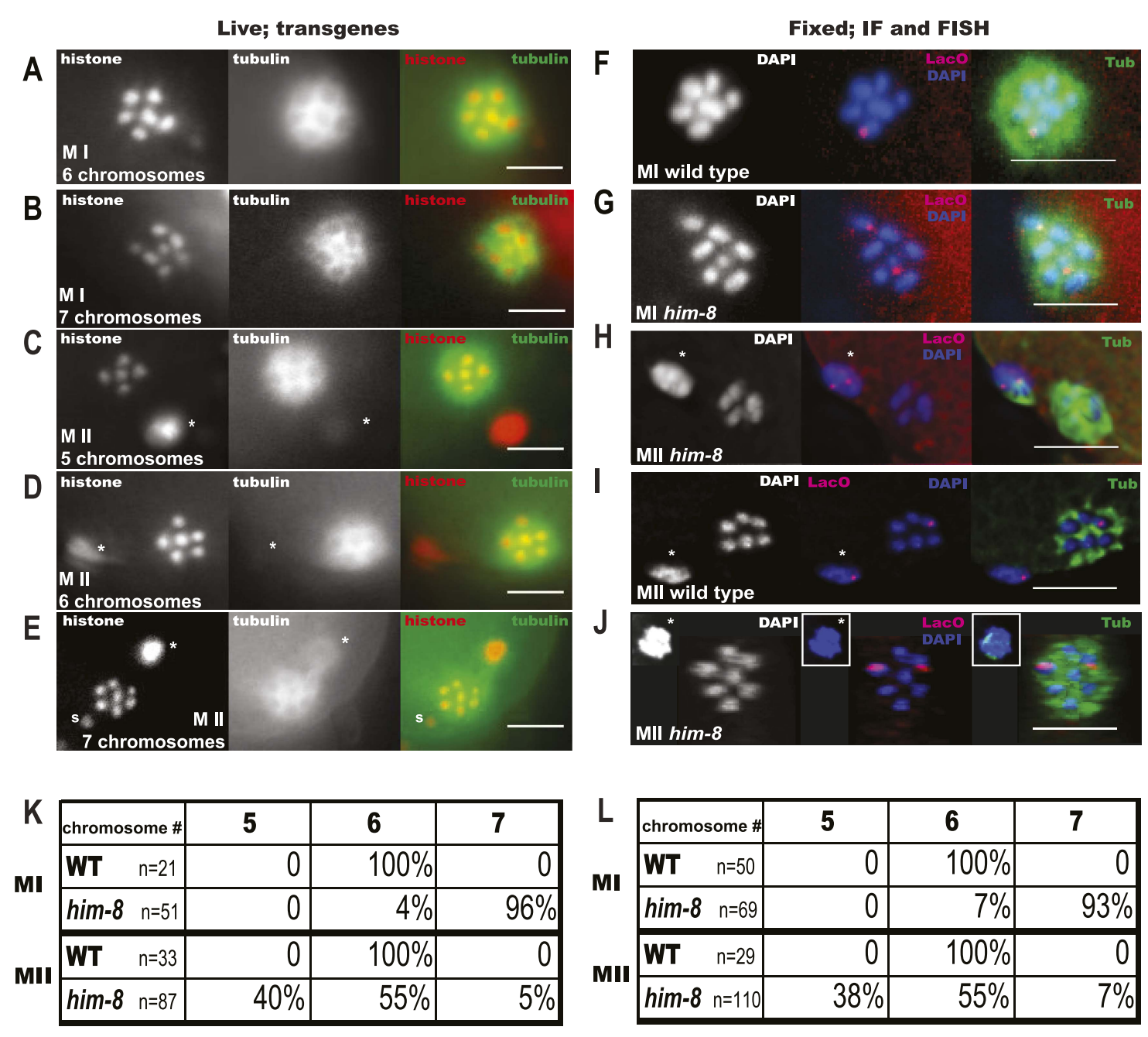

\begin{tabular}{|c|c|c|c|}
\hline chromosome \# & 5 & 6 & 7 \\
\hline WT $\quad n=50$ & 0 & $100 \%$ & 0 \\
\hline him-8 $n=69$ & 0 & $7 \%$ & $93 \%$ \\
\hline$n=29$ & 0 & $100 \%$ & 0 \\
\hline him $-8 \mathrm{n}=110$ & $38 \%$ & $55 \%$ & $7 \%$ \\
\hline
\end{tabular}

Figure 2. X univalents are preferentially lost between metaphase I and metaphase II in him-8 mutants. Z projections of living (A-E) and fixed (F-J) C. elegans meiotic embryos viewed down the pole-to-pole spindle axis at metaphase I (A, B, F, G) or metaphase II (C, D, E, H, I, J). mCherry::Histone H2B and GFP.:tubulin label the chromosomes and spindle, respectively, in live embryos. Fixed embryos were stained with DAPI, anti-tubulin antibody, and a LacO FISH probe that recognizes a LacO array integrated on the $\mathrm{X}$ chromosome. Asterisks indicate polar bodies. Insets show polar bodies that did not fit in the image frame. In $(\mathbf{E})$, 's' denotes a sperm outside of the embryo. Percentages are shown for each outcome $(\mathbf{K}, \mathbf{L})$. Bar $=5 \mu \mathrm{m}$.

DOI: 10.7554/eLife.06056.004

The following figure supplement is available for figure 2:

Figure supplement 1. zim-2 embryos also deposit unpaired chromosome $\mathrm{V}$ univalents into the first polar body.

DOI: 10.7554/eLife.06056.005

diploX ova inferred by Hodgkin et al. (1979) and support the hypothesis that the majority of $X$ univalents are eliminated between metaphase I and metaphase II. These maternal chromosome counts are also unaffected by nulloX or diploX sperm that might contribute to phenotype-based progeny counts.

To confirm that the two chromosomes lost between these stages were indeed the $\mathrm{X}$ univalents, we used FISH with a lac operator probe to detect a multi-copy lac operator array integrated on the $X$ chromosomes in a him-8 background (Figure 2F-J). FISH revealed two X univalents and 7 total DAPIstaining bodies on $93 \%$ of all the him-8 metaphase I plates (Figure 2G,L). At metaphase II, FISH revealed at least two $X$ hybridization foci in the first polar body and none on the metaphase plate when the spindle had 5 DAPI-staining bodies (Figure $2 \mathrm{H}$ ). When $6 \mathrm{DAPI}$-staining bodies were present on the metaphase II plate, we always observed one $\mathrm{X}$ hybridization focus each on the metaphase plate 
and in the first polar body (Figure 2I). Finally, metaphase II embryos containing 7 DAPI-staining bodies had two $X$ hybridization foci on the metaphase plate and none in the first polar body (Figure 2J). Together, these results demonstrate that both achiasmate $X$ univalents are deposited into the first polar body in $40 \%$ of him- 8 embryos as compared with the $25 \%$ expected from random segregation.

To test whether achiasmate autosomes are also placed in the first polar body with higher than random frequency, we analyzed a strain with a lac operator array integrated on chromosome $\mathrm{V}$ and bearing a loss of function mutation in the him-8 homolog zim-2, which contributes to chromosome $V$ pairing (Phillips and Dernburg, 2006). Unlike the situation with him-8 and pairing of the $X$, redundancy between ZIM proteins may contribute to chromosome $\mathrm{V}$ pairing. Phillips and Dernburg reported only $72 \%$ of diakinesis oocytes with 7 rather than 6 DAPI-staining bodies in a zim-2 mutant, and our zim-2 strain with lacO(V) had only $62 \%$ of diakinesis oocytes with 7 DAPI-staining bodies (Figure 2-figure supplement 1F). FISH revealed two distinct chromosome $V$ hybridization foci and 7 DAPI-staining bodies on $41 \%$ of metaphase I spindles (Figure 2-figure supplement 1A,E). Starting with $41 \%$ achiasmate V's, random segregation should yield $10 \%$ of metaphase II embryos with both V's in the first polar body (25\% of $41 \%$ ). Instead, FISH revealed $27 \%$ of metaphase II embryos had five DAPI-staining bodies on the metaphase plate and chromosome $\mathrm{V}$ hybridization foci only in the first polar body (Figure 2-figure supplement 1B,E). Likewise, random segregation of achiasmate V's should yield $10 \%$ metaphase II spindles with 7 DAPI-staining bodies on the metaphase II spindle, two distinct chromosome $\mathrm{V}$ hybridization foci on the spindle, and none in the first polar body. Only 5\% of this embryo class was observed (Figure 2-figure supplement 1D,E). These frequencies are significantly different than those expected from random segregation (chi-square test, two-tailed $p<0.0002$ ). The discrepancy in the fraction of zim-2 oocytes with 7 DAPI-staining bodies at diakinesis vs metaphase I raises the possibility that chromosomes might be systematically undercounted in zim-2 metaphase I spindles (but not in wild-type, him-8, or XXX metaphase I spindles). If this is the case, the two $\mathrm{V}$ univalents must be positioned close together on the spindle because 0/57 zim-2 metaphase I plates with 6 DAPI-staining bodies had two widely spaced lacO(V) FISH foci and the deviation between expected and observed nulloV metaphase II spindles would be even greater. Two results strongly indicate that the same mechanisms acting on univalent X's in him-8 mutants also act on V univalents in the zim-2 mutant. First, the fivefold preponderance of metaphase II spindles with 5 DAPIstaining bodies over those with 7 DAPI-staining bodies is similar to him-8. Second, the presence of lacO FISH signal only in the first polar body of metaphase II embryos with 5 DAPI-staining bodies on the spindle is the same in him-8 and zim-2. Thus, achiasmate autosomes, like achiasmate $X$ chromosomes, are preferentially deposited into the first polar body.

\section{Univalents biorient at metaphase I and tend to lag during anaphase I}

To understand the mechanism by which univalent $X$ chromosomes are preferentially deposited in polar bodies, we examined their orientation and position in the spindle. Antibodies specific for the cohesin subunit, REC-8, label a cruciform on metaphase I bivalents (Figure 3A,B) and a single band on metaphase II chromosomes (Figure 3A,C). The single REC-8 bands on wild-type metaphase II chromosomes and on him-8 metaphase I univalents were both oriented perpendicular to the pole-topole axis of the spindle (Figure 3D), indicating that him-8X univalents biorient at metaphase I. him-8 worms expressing GFP::KNL-2, which labels the $C$. elegans cup-shaped meiotic kinetochores (Dumont et al., 2010), were also analyzed for biorientation and yielded the same conclusion as analysis by REC-8 antibody (Figure 3-figure supplement 1). We also examined the localization of GFP:AIR-2, the aurora B kinase that is essential for the loss of cohesion at anaphase I and which is loaded between homologs of wild-type bivalents in a chiasma-dependent fashion (Rogers et al., 2002). The fluorescence intensity of GFP::AIR-2 was 2.3 times higher on bivalents than univalents at metaphase I of him-8 embryos (Figure 3-figure supplement 2B,C). AIR-2 is normally re-loaded between sister chromatids at metaphase II. GFP::AIR-2 on metaphase II chromosomes was 1.7 times higher than on him-8 metaphase I univalents (Figure 3-figure supplement 2C), indicating that the reduced amount of AIR-2 on metaphase I univalents was not simply a consequence of the smaller size of a univalent relative to a bivalent. AIR-2 is required for the crossover dependent, prometaphase, partial removal of REC-8 from between homologs in a wild-type bivalent, an event proposed to be essential for loss of cohesion at anaphase I (Severson and Meyer, 2014). Consistent with the low levels of AIR-2, him-8 univalents had $1.7 \pm 0.3$ times the intensity of REC- 8 staining as the 
A

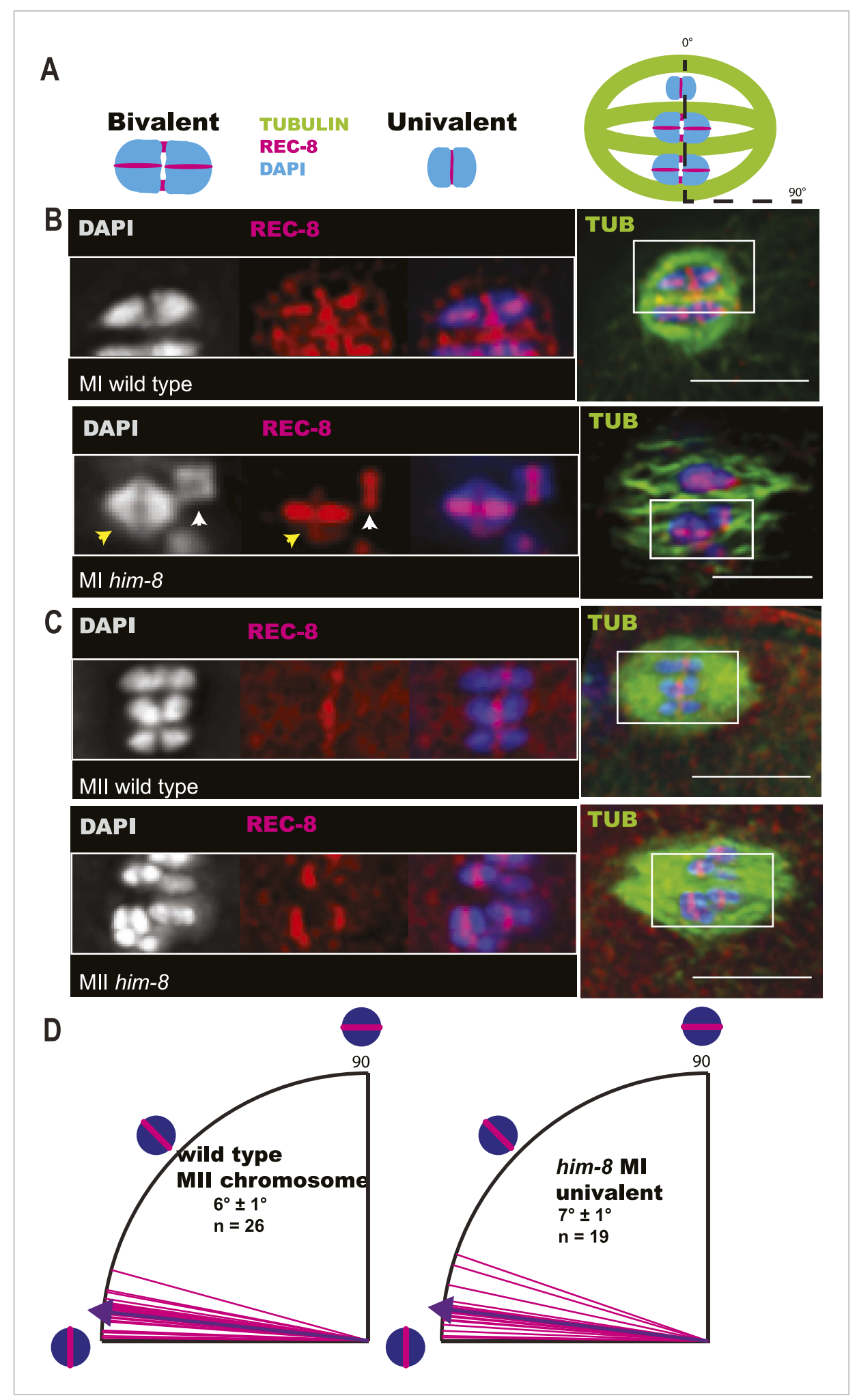

Figure 3. X univalents biorient at metaphase I in him-8 embryos. (A) Cartoon diagram of REC-8 staining on bivalents and univalents. (B and C) Anti-REC-8 staining of metaphase I and metaphase II embryos with bivalents (yellow arrow head) and univalents (white arrow head). In him-8 embryos, univalents at metaphase I have a single band of REC-8 with the same orientation seen on normal chromosomes at metaphase II. (D) Quantification of the orientation of Figure 3. continued on next page 
Figure 3. Continued

univalents by offset angle from the metaphase plate, $0^{\circ}$ corresponds to perfect biorientation and $90^{\circ}$ corresponds to perfect mono-orientation. Cortical pole is on the left in all images. Bar $=5 \mu \mathrm{m}$.

DOI: 10.7554/eLife.06056.006

The following figure supplements are available for figure 3:

Figure supplement 1. Imaging of GFP::KNL-2 demonstrates that him-8 univalent chromosomes biorient at metaphase of meiosis I.

DOI: 10.7554/eLife.06056.007

Figure supplement 2. Reduced levels of AIR-2 are loaded on him-8 X univalents at meiosis I. DOI: 10.7554/eLife.06056.008

inter-homolog region of bivalents in the same spindle (Figure $3 B ; n=8$ embryos, two-tailed $p=0.04$, chi square relative to expected 1.0).

Because $X$ univalents biorient at metaphase I but load half as much AIR-2, which is required for loss of cohesion at anaphase I in C. elegans (Kaitna et al., 2002; Rogers et al., 2002), and retain twice as much REC-8, we hypothesized that bioriented univalents might be pulled toward both spindle poles and lag on the anaphase spindle as they fail to lose cohesion. To test this possibility, we did time-lapse imaging of him-8 embryos expressing GFP::tubulin and mCherry::histone, focusing specifically on the events of anaphase I. $90 \%$ of him- 8 embryos at anaphase I had one or two lagging chromosomes $(n=119)$ compared to $2 \%$ of wild-type embryos $(n=52)$ (Figure $4 A, B)$. In $51 \%$ of living him- 8 embryos with lagging chromosomes at anaphase I, two discrete lagging chromosomes could be resolved. Each lagging chromosome eventually moved as a single unit either toward the cortex or into the embryo in $98 \%$ of embryos $(n=179$ ) (Figures $4 B, 5 A)$, indicating that cohesion between sister chromatids is maintained and that univalents are not broken or destroyed during anaphase. At anaphase II, only $10 \%$ of him- 8 embryos exhibited lagging chromosomes $(n=60)$ and $0 / 22$ wild-type embryos had lagging chromosomes, suggesting that lagging chromosomes are caused by the presence of univalents at meiosis I.

To confirm that the lagging chromosomes are bioriented $\mathrm{X}$ univalents, we used $\mathrm{LacO}(\mathrm{X}) \mathrm{FISH}$. We found that the X-specific FISH probe labeled one or two lagging chromosomes at anaphase I of him-8 embryos, indicating that lagging chromosomes are X univalents (13/14) (Figure 4D). 36\% of fixed him-8 anaphase I embryos with lagging chromosomes had two distinct FISH-positive chromosomes lagging. Another $21 \%$ had a single FISH-positive lagging body, but no other FISH-positive chromosomes on the spindle indicating that the two $\mathrm{X}$ univalents were likely too close to resolve in these embryos. The remaining $36 \%$ had one FISH-positive lagging chromosome and one FISH-positive chromosome in one of the main chromosome masses (one embryo had a lagging chromosome that was a bivalent). These results suggest that one or both X univalents lag in up to $90 \%$ of him- 8 embryos.

Similar results were obtained for chromosome $V$ in zim-2 mutants, where $40 \%$ of metaphase I embryos have univalent V's (Figure 2-figure supplement 1). 27\% (4/15 or over half of anaphase I spindles expected to have $V$ univalents) had a lagging chromosome. $100 \%$ (4/4) of these lagging chromosomes were chromosome $\mathrm{V}$ as assayed by LacO(V) FISH (Figure 4E). These results indicate that achiasmate autosomes lag at anaphase I, just like achiasmate $\mathrm{X}$ chromosomes.

\section{The meiotic contractile ring captures lagging $X$ univalents in the first polar body}

After establishing that lagging chromosomes were univalents, we next asked if univalents that lagged were subject to biased segregation at anaphase I. To analyze this, we conducted time-lapse imaging of embryos from him-8 worms expressing GFP::tubulin and mCherry::histone, as well as embryos from him-8 worms expressing these along with GFP::PH (plextrin homology domain) to label the plasma membrane (Figure 5A). Our time-lapse analysis revealed that $65 \%$ of lagging chromosomes eventually moved toward the cortex and the forming polar body of him-8 embryos during anaphase I $(n=181)$ (Figure 5B), indicating that preferential expulsion of lagging univalents into the first polar body could contribute to the higher than random frequency of metaphase II spindles with 5 autosomes and no X. Because the polar body contractile ring ingresses inward toward the midpoint of the late anaphase spindle where it normally scissions, we hypothesized that the preferential resolution of lagging chromosomes toward the cortex might result from inhibition of contractile ring scission 


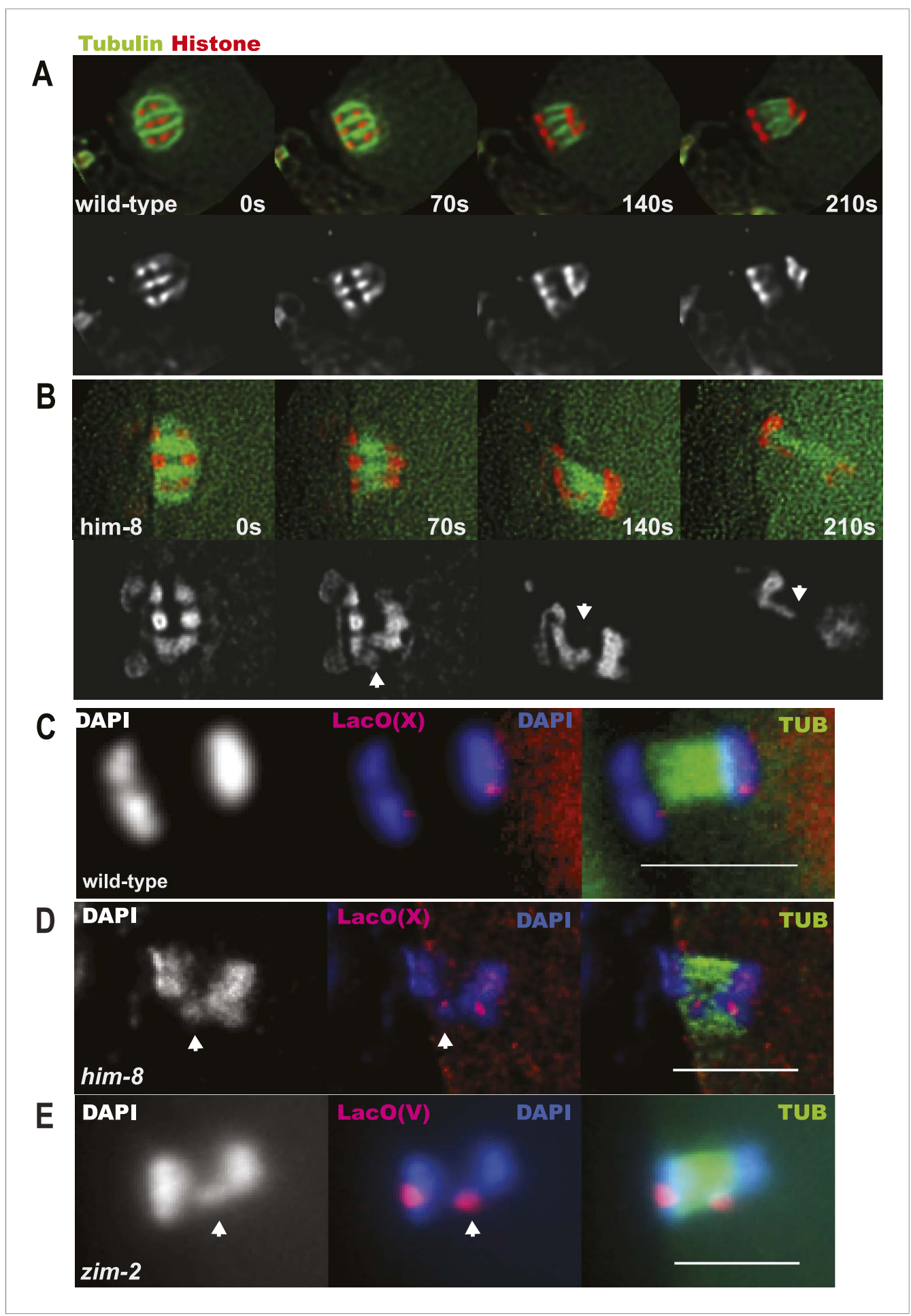

Figure 4. $X$ univalents lag at anaphase I. (A) Time-lapse images of a living wild-type embryo undergoing anaphase I show chromosomes separating as two distinct masses. (B) Time-lapse images of a living him-8 embryo show a lagging chromosome at anaphase I. (C-E) Z projections of fixed anaphase I embryos. (C) LacO FISH labeling of a wild-type strain with a $\mathrm{LacO}$ array integrated on the $\mathrm{X}$ chromosome shows normal segregation of two $\mathrm{X}$ homologs from one X bivalent. (D) LacO FISH shows that a lagging chromosome in him-8 is the X. (E) LacO FISH labeling of a zim-2 strain with a LacO array integrated on chromosome $V$ showing a univalent $V$ lagging at anaphase I. Cortical pole is to the left in all images. Bar $=5 \mu \mathrm{m}$.

DOI: 10.7554/eLife.06056.009 


\section{A}
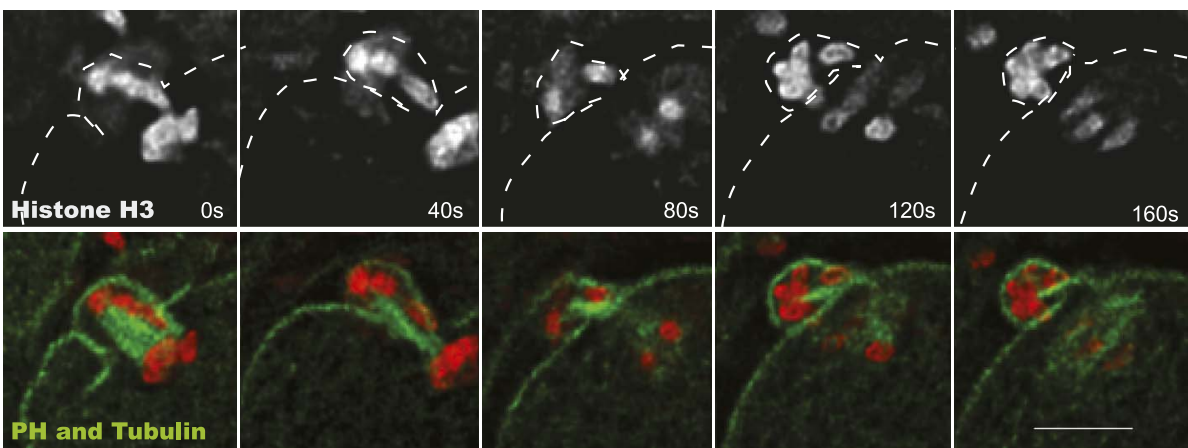

B

Contractile ring-dependent resolution bias of lagging chromosomes at anaphase I

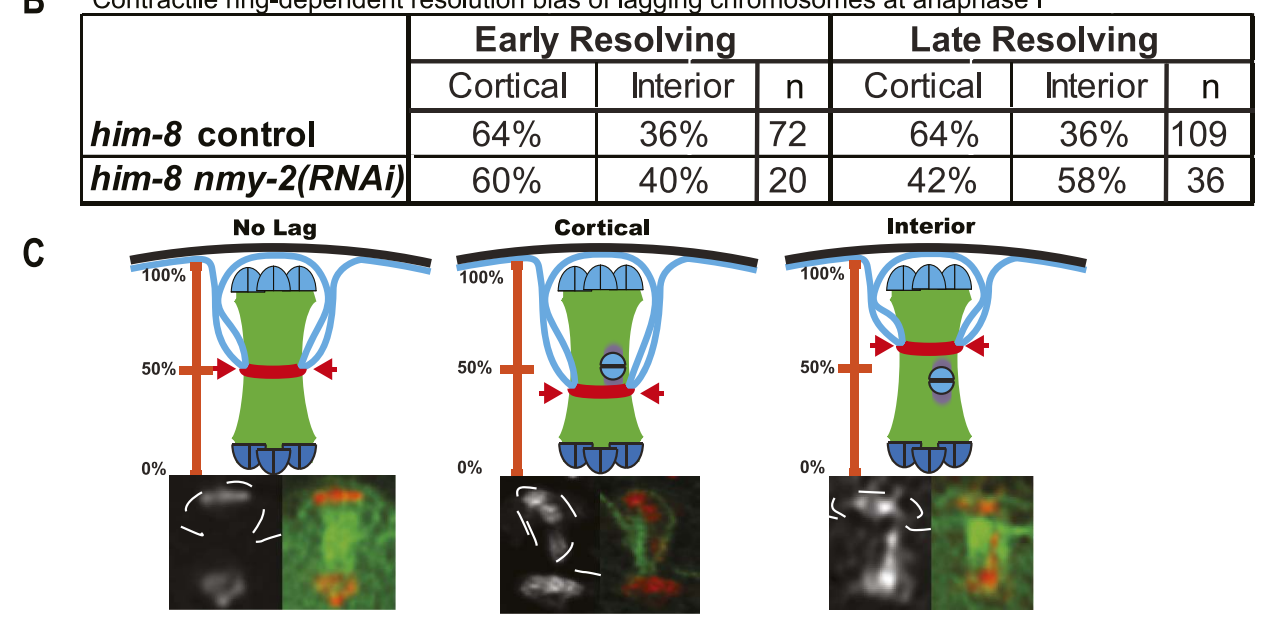

D

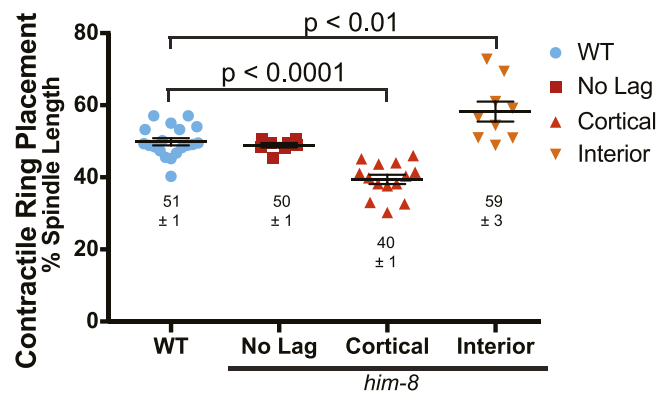

Figure 5. The contractile ring moves inward past the lagging chromosomes of him-8 embryos. (A) Time-lapse sequence of anaphase I in a him-8 strain with GFP::PH, GFP::Tubulin, and mCherry::Histone H2B. The plasma membrane ingresses past the lagging chromosomes to engulf them in the polar body. (B) Fraction of him-8 anaphase I embryos in which a lagging chromosome eventually resolved toward the cortex or eventually resolved into the embryo (interior). Lagging univalents resolved more frequently toward the cortex during both early and late anaphase. Depletion of NMY-2, the myosin required for polar body formation, eliminated only the late anaphase bias. Pairwise two-tailed $p$ values by Fisher's exact test: him-8 late vs him- $8 n m y-2\left(R N A_{i}\right)$ late $=0.02$, him- 8 early vs him-8 nmy-2(RNAi) early $=0.80$, him-8 early vs him-8 late $=1.0$, him-8 nmy-2(RNAi) early vs him-8 nmy-2(RNAi) late $=0.26$. $p$ values from Pearson's chi-squared test: him-8 late vs $50 \%=0.003$, him- 8 nmy-2(RNAi) late vs $50 \%=$ 0.32 , him -8 early vs $50 \%=0.02$, him -8 nmy-2(RNAi) early vs $50 \%=0.38$. (C) Top, diagram illustrating how the position of scission by the contractile ring along the pole-to-pole spindle axis was scored. Bottom, representative images from time-lapse sequences showing scission at different positions along the length of the spindle. (D) Average position of contractile ring scission along the pole-to-pole spindle axis in wild-type embryos and in him-8 embryos with no lagging chromosomes, lagging chromosomes that end up at the cortex (cortical), or lagging chromosomes that end up in the embryo (interior). Bar $=5 \mu \mathrm{m}$.

DOI: 10.7554/eLife.06056.010 
until the ring ingresses past lagging chromosomes (Figure 5C). In wild type, ingression of the polar body contractile ring initiates when homologs have separated by $2.3 \mu \mathrm{m}$ and polar body scission completes when homologs have separated by $5.6 \mu \mathrm{m}$ (Fabritius et al., 2011b). The bias of univalents that moved toward the cortex before initiation of contractile ring ingression could not be caused by engulfment by the polar body. Therefore, we separated lagging univalents into two categories, earlyresolving univalents that moved to one pole while the main chromosome masses were separated by less than $4.0 \mu \mathrm{m}$ and late-resolving univalents that moved to one pole only after the main chromosome masses were separated by greater than $4.0 \mu \mathrm{m}$. If late-resolving univalents were engulfed during polar body formation, elimination of contractile ring activity would reduce the fraction of lagging chromosomes resolving toward the cortex. Indeed, RNAi depletion of the non-muscle myosin, NMY-2, which causes complete loss of cortical furrowing and polar body formation (Fabritius et al., 2011b), in him-8 embryos resulted in a significant $(p=0.02)$ reduction in the percentage of late-lagging univalents resolving toward the cortex from $64 \%$ to $43 \%$ (Figure $5 B$ ).

As a complementary approach, we asked if more rapid polar body ring ingression would have the opposite effect of NMY-2 depletion. We previously showed that the depletion of the myosin phosphatase, MEL-11, doubled the rate of polar body ring ingression (Fabritius et alo, 2011b). Therefore, we hypothesized that the inactivation of MEL-11 might enhance the preferential engulfment of lagging univalents by the first polar body. Unlike NMY-2 depletion, which generates $100 \%$ dead embryos, the lethality of mel-11 mutants is rescued by wild-type sperm so the chromosome constitution of progeny from a mel-11 mother can be scored by phenotype. Mating otherwise wild-type males bearing the recessive X-linked marker, lon-2, to him-8 hermaphrodites allows measurement of the frequency of nulloX ova (which give rise to lon male progeny) and diploX ova (which give rise to XXX dumpy progeny) (Hodgkin et alo, 1979). Random segregation of univalents should generate a 1:1 ratio of nulloX:diploX ova. We found that mel-11 increased the segregation bias of him-8 by sevenfold from 3:1 to 23:1 (Table 1). This result indicates that more rapid furrow ingression captures more lagging univalents in the first polar body resulting in more nulloX ova.

Table 1. Enhancement of the segregation bias in him-8 mutants by mutations in the myosin phosphatase, mel-11

Self-progeny counts

\begin{tabular}{|c|c|c|c|c|c|}
\hline Genotype & Temperature $\left({ }^{\circ} \mathrm{C}\right)$ & $\%$ XO male & \% XX hermaphrodite & \% XXX Dpy & Total progeny \\
\hline mel-11(sb55) unc-4 & 20 & 0.2 & 99.8 & $\mathrm{NC}$ & 1763 \\
\hline mel-11(sb55) unc-4; him-8 & 20 & $49^{\star}$ & 51 & NC & 374 \\
\hline unc-4; him-8 & 20 & 34 & 66 & NC & 1442 \\
\hline mel-11(it126) unc-4 & 15 & 0.6 & 99 & NC & 790 \\
\hline mel-11(it126) unc-4; him-8 & 15 & $58^{\star}$ & 38.6 & 3.4 & 873 \\
\hline
\end{tabular}

Ratio of nulloX ova/diploX ova calculated from progeny of cross with lon-2 males

\begin{tabular}{|c|c|c|c|c|c|}
\hline Maternal genotype & Temperature $\left({ }^{\circ} \mathrm{C}\right)$ & \# NulloX (ion male progeny) & \# DiploX (dpy progeny) & Nullo/diplo & Total progeny \\
\hline mel-11(it26) unc-4 & 25 & 1 & 0 & NA & 785 \\
\hline mel-11(it26) unc-4; him-8 & 25 & 160 & 7 & 22.9 & 595 \\
\hline unc-4; him-8 & 25 & 98 & 31 & 3.2 & 677 \\
\hline
\end{tabular}

mel-11 increases the frequency of male progeny from him-8 mothers. mel-11(sb55) and mel-11(it26) worms produce high frequencies of dead embryos, which cannot be scored for sex at $25^{\circ} \mathrm{C}$ (Wissmann et al., 1999). Percent male $(X O)$, hermaphrodite $(X X)$, and dumpy $(X X X)$ progeny from self-fertilizing mel-11, him-8, or him-8 mel-11 double mutant worms were therefore scored at $15^{\circ} \mathrm{C}$ and $20^{\circ} \mathrm{C}$. Only progeny that developed to the $\mathrm{L} 4$ or adult stage were counted. ${ }^{*}$ Two-tailed $p<0.0001$ by binomial test compared with him- 8 alone. $100 \%$ of mel-11(it26) self progeny die as embryos at $25^{\circ} \mathrm{C}$, but this lethality is rescued by mel-11(+) sperm (Kemphues et al., 1988). The progeny of mel-11(it26) hermaphrodites crossed with lon-2 males could therefore be scored at $25^{\circ} \mathrm{C}$. When lon-2(+) hermaphrodites are crossed with lon-2 males (lon-2 is a recessive X-linked marker), $50 \%$ of the ova will be fertilized by sperm with a single lon- $2 \mathrm{X}$ chromosome. Fertilization of a nulloX ova by a lon-2X sperm will result in a lon-2 male. Fertilization of a diploX ova by a lon- $2 \mathrm{X}$ sperm will result in a XXX dumpy worm. Random segregation of the unpaired X chromosomes in him-8 would result in a ratio of nulloX/diplo X ova of 1.0 . The mel11; him-8 double mutant showed a sevenfold increase in the ratio of nullo/diploX ova relative to him-8 alone, indicating an increased efficiency of eliminating maternal unpaired $\mathrm{X}$ chromosomes.

DOI: 10.7554/eLife.06056.011 
To test our hypothesis that a lagging chromosome inhibits contractile ring scission to allow univalent capture, we asked whether the presence of late-lagging univalents might cause misplacement of the contractile ring from the $50 \%$ spindle length scission point observed in wildtype embryos (Fabritius et al., 2011b) by time-lapse imaging of the plasma membrane marker GFP:: $\mathrm{PH}$ (Figure 5A). Spindle length was measured between the outside edges of the main chromosome masses and only in frames in which both chromosome masses were in focus (Figure 5C). In him-8 embryos, when there were no lagging univalents, the contractile ring ingressed normally to $50 \%$ spindle length as measured from the outside edge of the main chromatin mass in the interior of the embryo. When a lagging univalent was seen segregating into the polar body, the contractile ring was seen ingressing deeper into the embryo to $40 \%$ spindle length (Figure $5 C, D$ ). Alternatively, when a lagging univalent was seen segregating into the embryo, the contractile ring ingressed inward to a shallower depth at $59 \%$ spindle length (Figure 5C,D).

To further test the idea that a late-lagging univalent might influence the choice of the scission point, we imaged formation of the first polar body in wild-type or him-8 worms expressing GFP:UNC59 (septin) and mCherry: histone. Septins are polymerizing GTPases that assemble in the contractile ring with myosin II, F-actin, and anillins (Green et al., 2013). In wild type or him-8 with early-resolving univalents, GFP:UNC-59 labeled a flat washer-shaped contractile ring that moved down to the midpoint of the elongating anaphase spindle as reported previously for GFP:NMY-2 (Fabritius et al., $2011 \mathrm{~b}$ ). The septin ring transformed into a tube (Figure 6A, $255 \mathrm{~s}$ ) as previously described for myosin and ANI-1 (Dorn et al., 2010). When cortical furrowing relaxed at the end of telophase I, the septin tube moved outward to the embryo surface, then flopped over, and remained as a separate entity next to the chromosomes in the first polar body (Figure 6A, $420 \mathrm{~s}$ ). In 5/10 him-8 embryos in which the septin-labeled ring reached the lagging univalent, the univalent was trapped in the septin tube and moved with the septin tube outward during cortical relaxation (Figure 6B). In these cases, the univalent remained trapped in the septin tube adjacent to the polar body as shown in Figure 6B, $C$. In 3/10 cases, the septin ring passed the univalent before the tube was formed, and in these cases, the univalent joined the main mass of chromatin in the polar body. In the 2/10 cases where the univalent did not end up in the polar body, the univalent slipped out of the septin tube into the embryo before the septin tube moved toward the embryo surface. These results are consistent with a model where the septin tube traps late-lagging univalents until scission occurs on the embryo side of the septin tube.

To further test the idea that late-lagging univalents are physically trapped in the septin tube, we tried to influence the integrity of the septin tube without blocking polar body scission. Septins act together with anillins (Green et al., 2013). C. elegans has three anillins: ANI-1 that is required for polar body scission, ANI-2 that is required for gonad development, and ANI-3 that has no known function (Maddox et al., 2005). We hypothesized that ANI-3 might play a non-essential structural role in the polar body septin tube and that ani-3(RNAi) might therefore allow late-lagging univalents to slip out of the tube back into the embryo. Indeed, RNAi of ANI-3 initiated on L4 him-8 hermaphrodites (which have already completed spermatogenesis) significantly ( $p<0.001$ binomial test) reduced the fraction of XO male progeny from $37 \%$ ( $n=9$ mothers, 1960 progeny) to $27 \%$ ( $n=11$ mothers, 2123 progeny), whereas ani-3 (RNAi) had no significant effect on wild-type worms (wt: $0.04 \% \mathrm{XO}, 1 \%$ dead, $\mathrm{n}=11$ mothers; ani-3 [RNAi]: 0.05\% XO, 1\% dead, $\mathrm{n}=17$ mothers). This result suggests that compromising the integrity of the septin tube may reduce the efficiency of trapping lagging univalents in the septin tube. ANI-3 depletion did not significantly increase the frequency of XXX dumpy progeny from him-8 mothers (him-8: 3\% XXX, 5\% dead; him-8 ani-3[RNAi]: 4\% XXX, 6\% dead). This apparent inconsistency might be explained if additional ANI-3-independent mechanisms act to reduce the number of XXX progeny (see below).

\section{The early anaphase segregation bias}

Lagging chromosomes were resolved prior to contractile ring ingression in $45 \%$ of embryos with lagging chromosomes at anaphase I. These were resolved toward the cortex $64 \%$ of the time $(n=72)$, and NMY-2 depletion had no significant effect on this class of embryos ( $p=0.8$ ) (Figure 5B). These results suggest that an additional mechanism biasing univalent movement toward the cortex might be at work earlier in the cell cycle. During wild-type meiosis, bivalents congress to the metaphase plate on an $8-\mu \mathrm{m}$ long spindle that is oriented parallel to the cortex. Upon anaphase promoting complex activation, the meiosis I spindle shortens to $4.8 \mu \mathrm{m}$ in length (Yang et al., 2003), then one spindle pole 


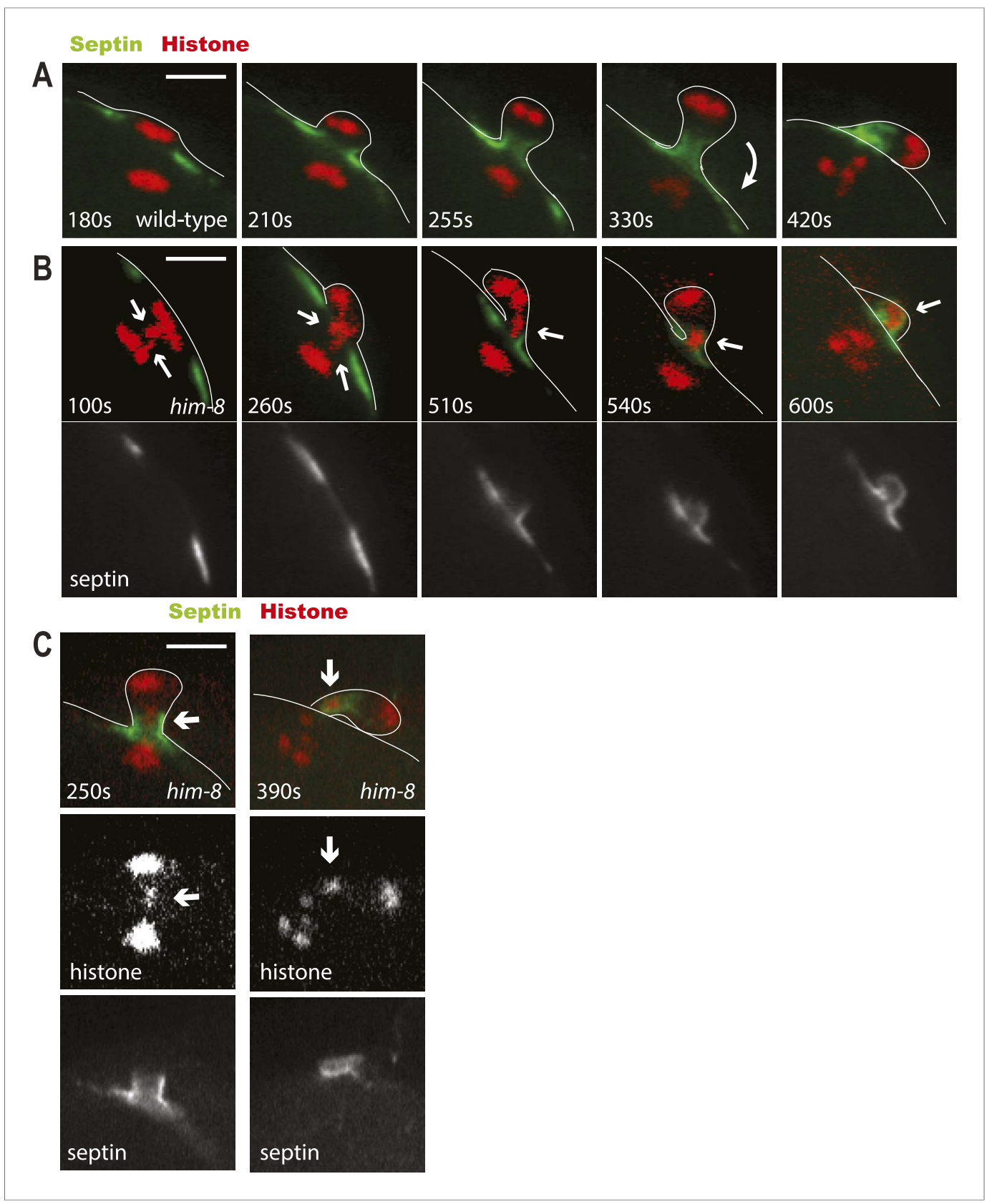

Figure 6. Lagging chromosomes are captured by the septin tube and expelled with polar bodies. Time-lapse imaging of embryos expressing GFP::septin and mCherry: histone. (A) Time-lapse images of a living wild-type embryo undergoing anaphase I show the conversion of a flat washer-shaped contractile ring into a tube during formation of the first polar body. (B) Time-lapse images of a living him-8 embryo show two lagging chromosomes at anaphase I (arrows) as one moves into the polar body early on, while the second is trapped in the septin tube and is extruded with the first polar body. (C) 2 time points of a him-8 embryo during telophase I showing the lagging chromosome trapped in the septin tube. Bar $=4 \mu \mathrm{m}$. Times are from the onset of homolog separation.

DOI: 10.7554/eLife.06056.012

moves to the cortex in a dynein-dependent manner, and homolog separation initiates (Ellefson and McNally, 2009, 2011). We found that univalents were misaligned toward the spindle poles in fixed him-8 embryos at late metaphase I, when the meiotic spindle is shortened but not yet rotated (Figure $7 A-B$ ). In $46 \%$ of these embryos, both univalents were misaligned toward the same pole (Figure 7B), close to the $50 \%$ expected from random positioning. In fixed him- 8 embryos at the onset of anaphase, when spindles are shortened and rotated but chromosomes are not yet separated, 57\% 


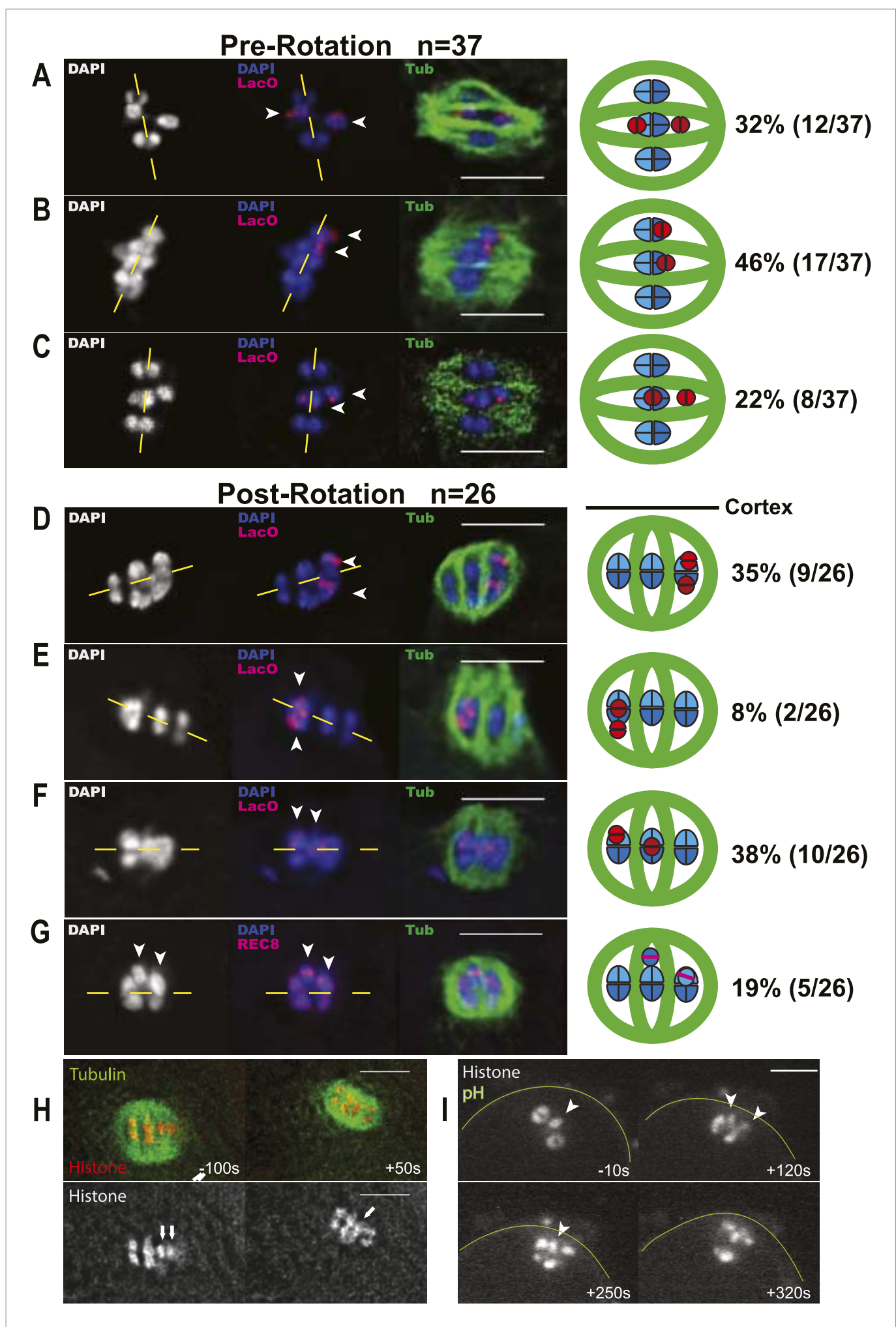

Figure 7. Early bias of univalent $X$ chromosomes might occur at the metaphase to anaphase $I$ transition. Representative cartoon diagrams and Z projections from fixed embryos stained with DAPI, anti-tubulin antibody, and $\mathrm{LacO}(\mathrm{X}) \mathrm{FISH}$ probe. Cortex is at the top. (A-C) Both $\mathrm{X}$ univalents on metaphase I spindles that were shortened (5.3-7.2 $\mu \mathrm{m}$ spindle length) but still parallel to the embryo cortex were frequently (46\%) aligned closer to the same spindle pole. (D-G) One or both univalents on Ml spindles that had rotated but homologs had not yet separated Figure 7. continued on next page 
Figure 7. Continued

were closer to the cortex and future polar body in $38+19 \%$ of embryos. Both univalents were never observed closer to the interior spindle pole. Yellow dashed lines indicate the metaphase plate. ( $\mathbf{H}$ and $\mathbf{I})$ Time-lapse images of two univalents (arrows in $\mathbf{H}$ ) or one univalent (arrowhead in $\mathbf{I}$ ) offset from the metaphase plate just before rotation of the univalent-proximal pole to the cortex. Time zero is initiation of spindle rotation. Bar $=5 \mu \mathrm{m}$. DOI: 10.7554/eLife.06056.013

had one or both univalents closer to the cortical pole $(38+19 \%$; Figure $7 F, G)$. No embryos had both univalents closer to the interior spindle pole. We hypothesized that one of two mechanisms might link spindle rotation with the early anaphase preference for univalent movement toward the cortex. Univalents might stochastically align closer to one spindle pole before rotation and bias the movement of that pole to the cortex. Alternatively, the cortex-proximal pole might acquire distinct biochemical properties after rotation due to cortical contact and subsequently generate more pulling force on the lagging univalents and pull them preferentially toward the cortex.

To test whether spindle rotation is involved with the him- 8 segregation bias, we utilized mei-2 (ct98), a partial loss of function katanin mutant, which causes a failure of meiotic spindle rotation but still allows polar body formation and production of viable progeny (McNally et al., 2006). If offset univalents bias spindle rotation or if the cortex-proximal pole exerts greater pulling on univalents after rotation, then a mei-2(ct98) him-8 double mutant should have a reduced frequency of male progeny relative to him-8 alone. At $20^{\circ} \mathrm{C}$, the mei-2(ct98) him-8 double mutant produced only $21 \%$ male progeny ( $n=1440$ progeny from 14 parents), which is significantly less than the $36 \%$ male progeny produced by the him- 8 single mutant $(n=964$ progeny from 8 parents; $p<0.0001$ by one-tailed binomial test) and is significantly different than mei-2 (ct98) alone ( $0 \%$ males; $n=925$ progeny from 8 parents). The reduction in male progeny is unlikely to be due to effects on spermatogenesis, as sperm is unaffected by katanin-null mutants (Mains et al., 1990). This result is consistent with either spindle rotation-based models for the early anaphase segregation bias. The role of spindle rotation is not conclusive; however, since mei-2(ct98) meiotic spindles have other phenotypes besides spindle rotation failure (McNally et al., 2006).

Absolute distinction between the two possible rotation models would require unambiguous tracking of both univalents before, during, and after spindle rotation. This was not possible in any of 201 time-lapse sequences. In 8 particularly clear time-lapse sequences, one or both univalents could be identified unambiguously 10-100 s before initiation of spindle rotation. In 6/8 of these cases, the univalent-proximal pole rotated to the cortex (Figure $7, \mathbf{H}$ ). In 1/8 cases, the univalent-proximal pole rotated away from the cortex. In $1 / 8$ cases, the two univalents were offset to opposite poles both before and during rotation. If the cortical environment conferred a stronger pulling force on the cortical pole after rotation, then lagging univalents crossing the midpoint of the anaphase spindle should be common. Time-lapse imaging of spindles after rotation revealed that among 30 embryos in which one or two lagging chromosomes were already positioned closer to one pole at anaphase I onset and the lagging chromosome resolved early, the lagging chromosome resolved toward the pole that it was already close to in $80 \%$ of these embryos (data not shown). Among the $20 \%$ of embryos in which the lagging chromosome moved to the opposite pole after spindle rotation, the chromosome moved toward the cortex 3 times, toward the embryo 3 times, and in one instance, the two lagging chromosomes resolved to opposite poles. These observations are not consistent with a cortical pole that generates a stronger pulling force after rotation but instead favor the idea that the offset position of univalents before rotation increases the probability that the univalent-proximal pole will move to the cortex.

Two results suggested that additional factors might contribute to the overall inheritance of univalent $X$ chromosomes. Both ani-3(RNAi) and mei-2(ct98) reduced the frequency of male selfprogeny from him-8 worms without increasing the frequency of triploX self-progeny. We therefore tested whether aneuploid sperm might influence phenotypic outcomes by LacO(X) FISH on pronuclear stage embryos from self-fertilized him-8 mothers (not shown). Before pronuclear meeting, male pronuclei are distinguished from female pronuclei by the presence of sperm asters. We observed $90 \%$ haploX, $8 \%$ nulloX, and $2 \%$ diploX male pronuclei $(n=52)$. These values are significantly different than the $50 \%, 25 \%, 25 \%$ expected from random segregation (two-tailed $p<0.0001$ by chi square) and are similar to the frequencies obtained by Hodgkin et al. (1979) using genetic tests with sex-reversed 
him-8 XX males. $10 \%$ nulloX sperm thus make a small contribution to reducing the frequency of XXX self-progeny.

\section{Discussion}

Hodgkin et al. (1979) showed that $C$. elegans that are trisomic for the $X$ chromosome or that fail to form a chiasma between the normal two $X$ homologs have fewer trisomic offspring than expected from random segregation. Our results explain this phenomenon by demonstrating that two cellular pathways preferentially segregate $X$ univalents into the first polar body. Mechanisms reducing the frequency of trisomic offspring have not been investigated in other model organisms because in both mouse and Drosophila, animals with trisomy $X$ are not fertile (Schupbach et al., 1978; Tada et al., 1993), and there are no mutants, like him-8, that specifically block crossover formation on one specific chromosome in females. However, women with trisomy 21 or trisomy $X$ are often fertile and have been reported to have more than 50\% euploid offspring (Bovicelli et al., 1982; Neri, 1984; Ratcliffe et alo, 1991; Robinson et alo, 1991; Stewart et al., 1991). Triploid oysters provide a stronger example of apparent female-specific correction to a diploid state. Eggs produced by triploid females and fertilized with sperm from diploid males result in 57\% diploid, 31\% triploid, and $12 \%$ aneuploid offspring, whereas eggs produced by diploids and fertilized by sperm from triploids result in $15 \%$ diploid and $85 \%$ aneuploid offspring (Gong et al., 2004). Gaging the likelihood that the phenomenon described here for $C$. elegans might be relevant to trisomic humans or triploid oysters is complicated by two issues. In contrast with trisomic C. elegans, triploid oysters (Guo and Allen, 1994) and trisomic human oocytes sometimes form trivalent structures rather than a separate bivalent and univalent. Only $42-16 \%$ of diplotene oocytes from fetuses with trisomy 21 , trisomy 13 , or trisomy 18 exhibited a separate bivalent and univalent (Roig et al., 2005; Robles et al., 2007). It is difficult to predict the behavior of trivalents on the spindle. In addition, it is not clear whether a univalent present during anaphase I of a human or oyster oocyte would be more likely to move to one pole intact as in C. elegans or to separate equationally. We speculate that single chromatids resulting from equational separation of univalents at anaphase I could be subjected to asymmetric segregation at anaphase II. Our results suggest that any chromosome that lags during late anaphase might be prefentially expelled simply due to the conserved asymmetric nature of polar body formation.

There is one example where a univalent chromosome exhibits the opposite of the segregation bias reported here in C. elegans. In the $44-78 \%$ of oocytes from XO mice in which the univalent segregates intact at anaphase I, the univalent is preferentially retained in the embryo (Lemaire-Adkins and Hunt, 2000). This appears to be a difference between worms and mice rather than a difference between a trisomy and a monosomy since sex-reversed XO C. elegans produce an excess of nulloX ova (Hodgkin, 1980).

Discerning the overall significance of preferentially placing univalents into the first polar body is a complex problem. In the case of an XXX mother or a mother with a mosaic ovary containing trisomic and diploid oocytes, these pathways would increase the frequency of normal haploid eggs relative to that expected from random distribution of a single univalent (Figure 1). In the case of diploid oocytes with two univalent autosomes, however, $100 \%$ efficient expulsion of univalents into the first polar body would increase the frequency of lethal monosomy. Data shown in Figure 2, however, show no significant decrease in haploid eggs (interpreted from the frequency of MII spindles with 6 chromosomes) from him-8 or zim-2 mothers relative to the $50 \%$ that would occur by random distribution. Thus, the efficiency of placing univalents in the first polar body has evolved to a point that corrects trisomy without reducing the frequency of haploid eggs produced from oocytes that failed to form a chiasma between one pair of homologs. The conservation of these mechanisms in other species will have to be elucidated by studies focused specifically on the concept of chromosomal errors that are corrected, rather than caused, by female meiotic spindles.

\section{Materials and methods}

\section{Worm strains}

The genotypes of $C$. elegans strains used in this work are listed in Supplementary file 1. For LacO(X) FISH, EG7477, which has lac operator arrays integrated on chromosome II and X, was outcrossed to him-8 males or to wild-type males to eliminate the extra LacO array on chromosome II, generating strains FM299 (wild-type $\mathrm{LacO}(\mathrm{X})$ ) and FM300 (him-8 lacO(X)). The loss of the chromosome II LacO array and homozygosity for the $\mathrm{X}$ chromosome array were confirmed by PCR. 


\section{RNAi}

RNAi was carried out by feeding bacteria (HT115) induced to express double-stranded RNA (Timmons et al., 2001). The clones used were nmy-2 I-3L24, ani-3 V-12J23 (Kamath et al., 2001).

\section{Live imaging}

Adult hermaphrodites were anesthetized with tricaine and tetramisole and immobilized between a coverslip and agarose pad on a slide. The time-lapse images shown in Figure $2 A-E$ and Figure 4A-B were captured on an Olympus (Center Valley, PA) IX71 microscope equipped with a 60X PlanApo NA 1.42 oil objective and an ORCA R2 CCD camera (Hamamatsu Photonics, Hamamatsu City, Japan). Hg arc excitation light was shuttered by a Sutter Lambda 10-3 shutter controller (Sutter Instruments, Novato, CA). Images shown in Figure 5A were captured with an Intelligent Imaging Innovations (Denver, CO) Marianas Spinning Disk Confocal equipped with a Photometrics (Tucson, AZ) Cascade QuantEM 512SC EMCCD, and Zeiss $63 \times 1.4$ objective. Image sequences in Figure 6 were captured with a Perkin Elmer-Cetus (Waltham, MA) Ultraview Spinning Disk Confocal equipped with an Orca R2 CCD and an Olympus 60× 1.4 objective.

\section{Immunofluorescence}

Meiotic embryos were extruded from hermaphrodites by gentle squishing between coverslip and slide, flash frozen in liquid $\mathrm{N}_{2}$, permeabilized by removing the coverslip, and then fixed in cold methanol before staining with antibodies and DAPI. Antibodies used in this work were mouse monoclonal anti-tubulin (DM1alpha, 1:200; Sigma), mouse monoclonal DM1alpha:FITC conjugated (1:30; Sigma), rabbit antiREC-8 (from 1:500; Josef Loidl), Alexa 594 anti-rabbit, and Alexa 594 anti-mouse (both from Molecular Probes and used at 1:200). Images in Figure 3 were captured with an Applied Precision Deltavision Deconvolution system equipped with an Olympus PlanApo 60× 1.40 objective and a CoolSnap HO CCD camera (Photometrics). Deltavision z-stacks were captured at 130-nm intervals. Images in Figures 1, 2F-J, 4C-E, 7 and Figure 2-figure supplement 1 were captured with the Olympus IX71 described above but using an Olympus DSU (disc scanning unit). Z stacks were acquired by taking images every 200 $\mathrm{nm}$ (unless otherwise noted) from the top to the bottom of the spindle tubulin signal.

\section{Deconvolution}

Deconvolution was performed on most images shown. Deconvolution of time-lapse movies from the IX71 was performed using Huygens Professional X11 (Scientific Volume Imaging, Hilversum, Netherlands), with point spread functions determined from bead images. Deltavision z-stacks were deconvolved using Softworx native deconvolution software, with PSFs calculated from bead images taken on that system.

\section{IF-C-FISH: immunofluorescence with chromosome FISH}

A lac operator oligonucleotide CCACATGTGGAATTGTG AGCGGATAACAATTTGTGG and an oligonucleotide corresponding to an X-specific repeat, XC (Phillips et al., 2005) TTTCGCTTAGAGC GATTCCTTACCCTTAAATGGGCGCCGG, were each synthesized with 3' and 5' Texas Red and used in hybridization to LacO arrays integrated on $\mathrm{X}$ or $\mathrm{V}$ or to endogenous $\mathrm{X}$ sequences. FISH with immunofluorescence was performed as described by Phillips et al. (2009) with some modifications.

Worms were washed in $0.8 \%$ egg buffer and then placed on slides pre-coated with poly-L-lysine (Sigma). Worms were then gently crushed between the slide and a $25-\mathrm{mm}$ sq. \#1 coverslip to extrude meiotic embryos and immediately submerged in liquid nitrogen for 10-15 min. Coverslips were then flicked off to freeze-crack eggshells, and slides were submerged in $-20^{\circ} \mathrm{C}$ methanol for $20-30 \mathrm{~min}$. Slides were then washed in 1x phosphate buffered saline (PBS) twice for $10 \mathrm{~min}$ and then in $1 \times$ PBST (PBS with $0.2 \%$ Tween-20) for $10 \mathrm{~min}$. Slides were then blocked in $1 \times$ PBST with $4 \%$ bovine serum albumen (BSA) for 30-45 min at room temperature in a moist chamber. Blocking solution was wicked away being careful not to dry out the samples, and FITC-conjugated DM1a was applied 1:30 in 1× PBST with 4\% BSA using $20 \mu$ l coverwells (Grace BioLabs). Slides were incubated in this antibody for $4 \mathrm{hr}$ at room temperature or left overnight at $4^{\circ} \mathrm{C}$. Slides were then washed sequentially in $1 \times$ PBST, $1 \times$ PBS, and $2 \times$ SSCT (saline sodium citrate buffer with $0.4 \%$ Tween-20) for $10 \mathrm{~min}$ each. Following the last wash, slides underwent secondary fixation in $7 \%$ formaldehyde in $1 \times$ egg buffer for 5 min and were immediately dipped in $2 \times$ SSCT to wash off fixative. Slides were then washed in $2 \times$ SSCT twice for 5 min 
each and then pre-hybridized. Pre-hybridization was performed by adding $200 \mu$ of $50 \%$ formamide in $2 \times \mathrm{SSCT}$ with a $200 \mu \mathrm{l}$ coverwell (Grace BioLabs) overnight at $37^{\circ} \mathrm{C}$ in a moist chamber. After $24 \mathrm{hr}$, slides were taken out of $37^{\circ} \mathrm{C}$ incubation and placed at room temperature while the FISH probe was prepared. The FISH probe was prepared by adding $0.6 \mu \mathrm{l}$ of the stock $(900 \mathrm{ng} / \mu \mathrm{l})$ to $30 \mu \mathrm{l}$ of hybridization buffer (hybridization buffer was made as described in Phillips et al., 2009) with $300 \mu \mathrm{l} / \mathrm{ml}$ salmon sperm DNA and $0.1 \%$ Tween-20 per slide. Slides were then incubated in $30 \mu \mathrm{l}$ of this solution under a hybridization slip (Grace BioLabs) at $95^{\circ} \mathrm{C}$ for $3 \mathrm{~min}$ on an OmniSlide (Thermo Scientific) and then at $37^{\circ} \mathrm{C}$ in a moist chamber for 48-72 hr. Following this incubation, slides were washed in 50\% formamide in $2 \times$ SSCT as before but for two 1-hr incubations. Finally, slides were stained with DAPI by submerging in a Coplin jar filled with $2 \times$ SSCT $6 \mu \mathrm{g} / \mathrm{ml}$ DAPI for $10 \mathrm{~min}$ and were then washed for $30 \mathrm{~min}$ in fresh $2 \times$ SSCT. Slides were then wicked dry with a Kimwipe taking care not to dry out the sample and were mounted with $8 \mu \mathrm{l}$ of DABCO Mowiol and sealed with nail polish. Following 2-3 days for curing, slides were imaged.

\section{Metaphase chromosome counts}

Chromosome counts were carried out on live embryos in utero or on fixed embryos extruded from the worm by locating metaphase spindles whose chromosomes were all aligned at the metaphase plate. Z stacks were captured at $200-\mathrm{nm}$ intervals. Spindles that were oriented sideways, with their metaphase plates perpendicular to the imaging plane, were reconstructed using ImageJ 3D stack reconstruction, and chromosomes were counted only if individual masses could be discerned. Metaphase II spindles were distinguished from metaphase I spindles by the presence of polar bodies.

\section{Analysis of lagging chromosome resolution}

Time-lapse images of lagging chromosomes in FM125, FM126, and FM232 were acquired at 10-s intervals beginning at late metaphase I when the spindle is shortening and rotating and continuing through polar body extrusion and the formation of the metaphase II spindle. The direction of resolution was determined from the last frame where the lagging chromosome was still discernable from the segregating chromosome masses. At this frame, spindle length was determined by measuring the distance between the outside edges of the main masses of segregating chromosomes. Lagging chromosomes that decided which way to go when the spindle was more than $4 \mu \mathrm{m}$ long were classified as late resolving because earlier work indicated that myosin-dependent polar body scission occurs when spindles are longer than $4 \mu \mathrm{m}$ (Fabritius et al., 2011b). We confirmed this assumption by finding that 5/5 FM232 (GFP:PH) spindles longer than $4 \mu \mathrm{m}$ exhibited deep cortical furrows.

For nmy-2(RNAi) time-lapse sequences, only embryos in which polar body extrusion completely failed were analyzed. The fate of lagging chromosomes was scored based on whether they ended up at the cortex or in the interior prior to the formation of the metaphase II spindle. Often, chromosomes at the cortex were picked up by the metaphase II spindle. These were still scored as cortex-fated lagging chromosomes.

\section{Acknowledgments}

We thank the following for Caenorhabditis elegans strains: Christian Frøkjær-Jensen, Erik Jorgensen, Aaron Severson, Barbara Meyer, Anne Villeneuve, Amy Maddox, Becky Green, Karen Oegema, Paula Checci, and the Caenorhabditis Genetics Center, which is funded by the NIH Office of Research Infrastructure Programs (P40 OD010440). This work was supported by National Institute of General Medical Sciences Grant 1R01GM-079421 (to FJM), a grant from the Canadian Institutes of Health Research (To PEM), a fellowship from the Floyd and Mary Schwall (to DC), and NIH training grant T32 GM007377 (to DC). We also thank Dan Starr, JoAnne Engebrecht, Amy Fabritius, Lesilee Rose, and Jon Flynn for critical reading of the manuscript.

\section{Additional information}

Funding

\begin{tabular}{lll} 
Funder & Grant reference & Author \\
\hline $\begin{array}{l}\text { National Institute of General } \\
\text { Medical Sciences (NIGMS) }\end{array}$ & 1R01GM-079421 & $\begin{array}{l}\text { Francis J } \\
\text { McNally }\end{array}$ \\
\hline $\begin{array}{l}\text { Canadian Institutes of Health } \\
\text { Research }\end{array}$ & $\begin{array}{l}\text { Title: Genetic analysis of the } \\
\text { C. elegans cytoskeleton }\end{array}$ & Paul E Mains \\
\hline
\end{tabular}




\begin{tabular}{lll} 
Funder & Grant reference & Author \\
\hline $\begin{array}{l}\text { National Institutes of Health } \\
\text { (NIH) }\end{array}$ & Training Grant: T32 GM007377 & Daniel B Cortes \\
\hline University of California, Davis & $\begin{array}{l}\text { Floyd and Mary Schwall } \\
\text { fellowship }\end{array}$ & Daniel B Cortes \\
\hline $\begin{array}{l}\text { National Institutes of Health } \\
\text { (NIH) }\end{array}$ & $\begin{array}{l}\text { Office of Research Infrastructure } \\
\text { Programs P40 OD010440 }\end{array}$ & $\begin{array}{l}\text { Francis J } \\
\text { McNally }\end{array}$ \\
\hline
\end{tabular}

The funders had no role in study design, data collection and interpretation, or the decision to submit the work for publication.

Author contributions

DBC, KLMN, PEM, Conception and design, Acquisition of data, Analysis and interpretation of data, Drafting or revising the article; FJMN, Conception and design, Analysis and interpretation of data, Drafting or revising the article

\section{Additional files}

Supplementary files

- Supplementary file 1. C. elegans strains used in this study.

DOI: 10.7554/eLife.06056.014

- Supplementary file 2. Z-stack of XC FISH on XXX wild-type metaphase plate in meiosis I. 16-bit 3-channel TIFF can be opened using FIJI or basic ImageJ (http://fiji.sc/Downloads). Data shown are a z-stack acquired with $300 \mathrm{~nm}$ steps through a meiosis I metaphase spindle. Chromosomes are shown in blue (DAPI), tubulin antibodies label the spindle in green, and the XC FISH probe labels X chromosomes (2 present) in red. Channels can be split for individual analysis using the channel splitter (Image > Colors > Split channels) or can be hidden using the channels tool (Image > Colors > Channels tool).

DOI: 10.7554/eLife.06056.015

- Supplementary file 3. Z-stack of XC FISH on XXX wild-type metaphase plate in meiosis II. 16-bit 3-channel TIFF can be opened using FIJI or basic ImageJ (http://fiji.sc/Downloads). Data shown are a z-stack acquired with $300 \mathrm{~nm}$ steps through a meiosis II metaphase spindle. Chromosomes and the first polar body, which is on the top, are shown in blue (DAPI), tubulin antibodies label the spindle in green, and the XC FISH probe labels X chromosomes (1 present on the spindle) in red. Channels can be split for individual analysis using the channel splitter (Image > Colors $>$ Split channels) or can be hidden using the channels tool (Image > Colors > Channels tool).

DOI: 10.7554/eLife.06056.016

\section{References}

Bhalla N, Dernburg AF. 2005. A conserved checkpoint monitors meiotic chromosome synapsis in Caenorhabditis elegans. Science 310:1683-1686. doi: 10.1126/science.1117468.

Bovicelli L, Orsini LF, Rizzo N, Montacuti V, Bacchetta M. 1982. Reproduction in Down syndrome. Obstetrics and Gynecology 59(6 Suppl):13S-17S.

Buonomo SB, Clyne RK, Fuchs J, Loidl J, Uhlmann F, Nasmyth K. 2000. Disjunction of homologous chromosomes in meiosis I depends on proteolytic cleavage of the meiotic cohesin Rec8 by separin. Cell 103:387-398. doi: 10. 1016/S0092-8674(00)00131-8.

Checchi PM, Lawrence KS, Van MV, Larson BJ, Engebrecht J. 2014. Pseudosynapsis and decreased stringency of meiotic repair pathway choice on the hemizygous sex chromosome of Caenorhabditis elegans males. Genetics 197:543-560. doi: 10.1534/genetics.114.164152.

Dorn JF, Zhang L, Paradis V, Edoh-Bedi D, Jusu S, Maddox PS, Maddox AS. 2010. Actomyosin tube formation in polar body cytokinesis requires Anillin in C. elegans. Current Biology 20:2046-2051. doi: 10.1016/j.cub.2010.10.030.

Dumont J, Oegema K, Desai A. 2010. A kinetochore-independent mechanism drives anaphase chromosome separation during acentrosomal meiosis. Nature Cell Biology 12:894-901. doi: 10.1038/ncb2093.

Ellefson ML, McNally FJ. 2009. Kinesin-1 and cytoplasmic dynein act sequentially to move the meiotic spindle to the oocyte cortex in Caenorhabditis elegans. Molecular Biology of the Cell 20:2722-2730. doi: 10.1091/mbc. E0812-1253.

Ellefson ML, McNally FJ. 2011. CDK-1 inhibits meiotic spindle shortening and dynein-dependent spindle rotation in C. elegans. The Journal of Cell Biology 193:1229-1244. doi: 10.1083/jcb.201104008. 
Fabritius AS, Ellefson ML, McNally FJ. 2011a. Nuclear and spindle positioning during oocyte meiosis. Current Opinion in Cell Biology 23:78-84. doi: 10.1016/j.ceb.2010.07.008.

Fabritius AS, Flynn JR, McNally FJ. 2011b. Initial diameter of the polar body contractile ring is minimized by the centralspindlin complex. Developmental Biology 359:137-148. doi: 10.1016/j.ydbio.2011.08.013.

Frøkjær-Jensen C, Davis MW, Sarov M, Taylor J, Flibotte S, LaBella M, Pozniakovsky A, Moerman DG, Jorgensen EM. 2014. Random and targeted transgene insertion in Caenorhabditis elegans using a modified Mos1 transposon. Nature Methods 11:529-534. doi: 10.1038/nmeth.2889.

Goldstein P. 1984. Triplo-X hermaphrodite of Caenorhabditis elegans: pachytene karyotype analysis, synaptonemal complexes, and pairing mechanisms. Canadian Journal of Genetics and Cytology 26:13-17. doi: 10.1139/g84-003.

Gong N, Yang H, Zhang G, Landau BJ, Guo X. 2004. Chromosome inheritance in triploid Pacific oyster Crassostrea gigas Thunberg. Heredity 93:408-415. doi: 10.1038/sj.hdy.6800517.

Green RA, Mayers JR, Wang S, Lewellyn L, Desai A, Audhya A, Oegema K. 2013. The midbody ring scaffolds the abscission machinery in the absence of midbody microtubules. The Journal of Cell Biology 203:505-520. doi: 10. 1083/jcb.201306036.

Guo X, Allen SK. 1994. Reproductive potential and genetics of triploid pacific oysters, Crassostrea gigas (Thunberg). The Biological Bulletin 187:309-318. doi: 10.2307/1542288.

Hodgkin J. 1980. More sex-determination mutants of Caenorhabditis elegans. Genetics 96:649-664.

Hodgkin J, Horvitz HR, Brenner S. 1979. Nondisjunction mutants of the Nematode Caenorhabditis elegans. Genetics 91:67-94.

Kaitna S, Pasierbek P, Jantsch M, Loidl J, Glotzer M. 2002. The aurora B kinase AIR-2 regulates kinetochores during mitosis and is required for separation of homologous chromosomes during meiosis. Current Biology 12:798-812. doi: 10.1016/S0960-9822(02)00820-5.

Kamath RS, Martinez-Campos M, Zipperlen P, Fraser AG, Ahringer J. 2001. Effectiveness of specific RNAmediated interference through ingested double-stranded RNA in Caenorhabditis elegans. Genome Biology 2: RESEARCH0OO2.

Kemphues KJ, Kusch M, Wolf N. 1988. Maternal-effect lethal mutations on linkage group II of Caenorhabditis elegans. Genetics 120:977-986.

Kouznetsova A, Lister L, Nordenskjöld M, Herbert M, Höög C. 2007. Bi-orientation of achiasmatic chromosomes in meiosis I oocytes contributes to aneuploidy in mice. Nature Genetics 39:966-968. doi: 10.1038/ng2065.

Lemaire-Adkins R, Hunt PA. 2000. Nonrandom segregation of the mouse univalent X chromosome: evidence of spindle-mediated meiotic drive. Genetics 156:775-783.

Lewellyn L, Carvalho A, Desai A, Maddox AS, Oegema K. 2011. The chromosomal passenger complex and centralspindlin independently contribute to contractile ring assembly. The Journal of Cell Biology 193:155-169. doi: 10.1083/jcb.201008138.

Maddox AS, Azoury J, Dumont J. 2012. Polar body cytokinesis. Cytoskeleton 69:855-868. doi: 10.1002/cm.21064.

Maddox AS, Habermann B, Desai A, Oegema K. 2005. Distinct roles for two C. elegans anillins in the gonad and early embryo. Development 132:2837-2848. doi: 10.1242/dev.01828.

Mains PE, Kemphues KJ, Sprunger SA, Sulston IA, Wood WB. 1990. Mutations affecting the meiotic and mitotic divisions of the early Caenorhabditis elegans embryo. Genetics 126:593-605.

Maro B, Verlhac MH. 2002. Polar body formation: new rules for asymmetric divisions. Nature Cell Biology 4: E281-E283. doi: 10.1038/ncb1202-e281.

McNally K, Audhya A, Oegema K, McNally FJ. 2006. Katanin controls mitotic and meiotic spindle length. The Journal of Cell Biology 175:881-891. doi: 10.1083/jcb.200608117.

McNally KL, Fabritius AS, Ellefson ML, Flynn JR, Milan JA, McNally FJ. 2012. Kinesin-1 prevents capture of the oocyte meiotic spindle by the sperm aster. Developmental Cell 22:788-798. doi: 10.1016/j.devcel.2012.01.010.

Miller MP, Amon A, Ünal E. 2013. Meiosis I: when chromosomes undergo extreme makeover. Current Opinion in Cell Biology 25:687-696. doi: 10.1016/j.ceb.2013.07.009.

Moore DP, Orr-Weaver TL. 1998. Chromosome segregation during meiosis: building an unambivalent bivalent. Current Topics in Developmental Biology 37:263-299.

Mlynarczyk-Evans S, Roelens B, Villeneuve AM. 2013. Evidence that masking of synapsis imperfections counterbalances quality control to promote efficient meiosis. PLOS Genetics 9:e1003963. doi: 10.1371/journal. pgen.1003963.

Neri G. 1984. A possible explanation for the low incidence of gonosomal aneuploidy among the offspring of triplo-X individuals. American Journal of Medical Genetics 18:357-364. doi: 10.1002/ajmg.1320180220.

Nicklas RB, Jones K. 1977. Chromosome distribution: experiments on cell hybrids in vitro. Philosophical Transactions of the Royal Society of London Series B, Biological Sciences 277:267-276. doi: 10.1098/rstb.1977. 0017.

Phillips CM, Dernburg AF. 2006. A family of zinc-finger proteins is required for chromosome-specific pairing and synapsis during meiosis in C. elegans. Developmental Cell 11:817-829. doi: 10.1016/j.devcel.2006.09.020.

Phillips CM, McDonald KL, Dernburg AF. 2009. Cytological analysis of meiosis in Caenorhabditis elegans. Methods in Molecular Biology 558:171-195. doi: 10.1007/978-1-60761-103-5_11.

Phillips CM, Wong C, Bhalla N, Carlton PM, Weiser P, Meneely PM, Dernburg AF. 2005. HIM-8 binds to the X chromosome pairing center and mediates chromosome-specific meiotic synapsis. Cell 123:1051-1063. doi: 10. 1016/j.cell.2005.09.035.

Ratcliffe SG, Butler GE, Jones M. 1991. Edinburgh study of growth and development of children with sex chromosome abnormalities. IV in children and young adults with sex chromosome aneuploidy. In: Evans JA, 
Hamerton JL, Robinson A, editors. March of dimes birth defects foundation birth efects original article series, volume 26. No.4, 1990.

Robinson A, Bender BG, Linden MG, Salbenblatt JA. 1991. Sex chromosome aneuploidy: the denver prospective study in children and young adults with sex chromosome aneuploidy. In: Evans JA, Hamerton JL, Robinson A, editors. March of Dimes birth defects Foundation birth efects original article Series, volume 26. No.4, 1990.

Robles P, Roig I, Garcia R, Ortega A, Egozcue J, Cabero LL, Garcia M. 2007. Pairing and synapsis in oocytes from female fetuses with euploid and aneuploid chromosome complements. Reproduction 133:899-907. doi: 10. 1530/REP-06-0243.

Roig I, Robles P, Garcia R, Martínez-Flores I, Cabero L, Egozcue J, Liebe B, Scherthan H, Garcia M. 2005. Chromosome 18 pairing behavior in human trisomic oocytes. Presence of an extra chromosome extends bouquet stage. Reproduction 129:565-575. doi: 10.1530/rep.1.00568.

Rogers E, Bishop JD, Waddle JA, Schumacher JM, Lin R. 2002. The aurora kinase AIR-2 functions in the release of chromosome cohesion in Caenorhabditis elegans meiosis. The Journal of Cell Biology 157:219-229. doi: 10. 1083/jcb.200110045.

Schupbach T, Wieschaus E, Nothinger R. 1978. A study of the female germline in mosaics of Drosophila. Wilhelm Roux's Archives of Developmental Biology 184:41-56.

Severson AF, Meyer BJ. 2014. Divergent kleisin subunits of cohesin specify mechanisms to tether and release meiotic chromosomes. eLife 3:e03467. doi: 10.7554/eLife.03467.

Stewart DA, Bailey JD, Netley CT, Park E. 1991. Growth, development, and behavioral outcome from midadolescence to adulthood in subjects with chromosome aneuploidy: the Toronto study. in children and young adults with sex chromosome aneuploidy. In: Evans JA, Hamerton JL, Robinson A, editors. March of dimes birth defects foundation birth efects original article series, volume 26. No.4, 1990.

Tada T, Takagi N, Adler ID. 1993. Parental imprinting on the mouse X chromosome: effects on the early development of X0, XXY and XXX embryos. Genetical Research 62:139-148. doi: 10.1017/S0016672300031736. Timmons L, Court DL, Fire A. 2001. Ingestion of bacterially expressed dsRNAs can produce specific and potent genetic interference in Caenorhabditis elegans. Gene 263:103-112. doi: 10.1016/S0378-1119(00)00579-5.

Wissmann A, Ingles J, Mains PE. 1999. The Caenorhabditis elegans mel-11 myosin phosphatase regulatory subunit affects tissue contraction in the somatic gonad and the embryonic epidermis and genetically interacts with the Rac signaling pathway. Developmental Biology 209:111-127. doi: 10.1006/dbio.1999.9242.

Yang HY, McNally K, McNally FJ. 2003. MEI-1/katanin is required for translocation of the meiosis I spindle to the oocyte cortex in C. elegans. Developmental Biology 260:245-259. doi: 10.1016/S0012-1606(03)00216-1. 